\title{
Photoacoustic Densitometry of Ovalbumin on Nitrocellulose Sheet by Imaging by Fast Laser Beam Scanning
}

\author{
Katsumi UchiYama*, Keiko OHSAWA*, Tetsuya Mita*, Yoshihiro Yoshimura**, \\ Kazuo IMAEDA** and Kohei TAMURA*** \\ *Department of Instrumental Analysis, Institute of Medical Chemistry, Hoshi University, \\ Ebara, Sinagawa, Tokyo 142, Japan \\ ** Department of Analytical Chemistry, Faculty of Pharmaceutical Science, Hoshi University, \\ Ebara, Sinagawa, Tokyo 142, Japan \\ *** Ohkura National Hospital, Setagaya, Tokyo 157, Japan
}

\begin{abstract}
Photoacoustic densitometry by using a fast laser beam with sinusoidal scanning was conducted. The signal distortion was improved by using a sinusoidal scanning system combined with a Bessel high-pass filter. However, small distortions were found to remain in the densitograms, compared with those produced by conventional light scanning. Ovalbumin was two-dimensionally electrophoresed, followed by blotting on a nitrocellulose sheet. The signal intensity of ovalbumin on a nitrocellulose sheet was about 5-times greater than that on polyacrylamide gel. The reproducibility (RSD) of measurements for ovalbumin on four different nitrocellulose sheets was in the $8-15 \%$ range. However, the value was less than $5 \%$ for measurements of ovalbumin on the same plate. The detection limit of ovalbumin was about $150 \mathrm{ng}$. Ovalbumin, $1.67 \mu \mathrm{g}$ of which was spiked in calf serum, was almost recovered by the method.
\end{abstract}

Keywords Photoacoustic spectrometry, fast laser beam scanning, two dimensional gel electrophoresis, ovalbumin, nitrocellulose sheet

Polyacrylamide gel electrophoresis (PAGE) is a widely used separation method which depends on differences in isoelectric points ${ }^{1}$ and molecular weights ${ }^{2}$ of the constituent materials. PAGE plays significant roles in biological studies, e.g. isolation of a single protein by O'Farrell's two-dimensional electrophoresis ${ }^{3}$, PAGE combined immuno technique ${ }^{4}$, etc. The electrophoresed proteins on a gel can usually be visualized by staining them with acidic dyes or, exposing them to autoradiography when sample proteins are radiolabeled. The amounts of proteins on gels were determined either by a light reflection-type scanning densitometer ${ }^{5}$ or by the use of video camera densitometry. ${ }^{6}$

Moller el al. ${ }^{7}$ demonstrated the effectiveness and sensitivity of photoacoustic densitometry combined with isoelectric focusing followed by staining with Coomassie Blue. We have also reported on a lightscanning photoacoustic densitometer (s-PAS) ${ }^{8,9}$, which is applicable to any sample with a flat surface. We applied this apparatus to the determination of nanogram amounts of protein. ${ }^{10}$ The s-PAS showed high sensitivity to protein on silica-gel thin-layer chromatography (TLC) plates and on polyacrylamide gels. However, it took a long time to determine small amounts of samples since an alternate photoacoustic signal required a large conversion time to produce an exact photoacoustic densitogram for conventional size
TLC plates or electrophoresed gel plates.

We have also developed a photoacoustic densitometry method using fast laser beam scanning ${ }^{11}$ (FS-PAS). The densitogram could be obtained in less than a second by simple laser beam scanning. The signal-to-noise ratio $(S / N)$ of the densitogram could be increased by accumulating the analogue to digital (A/D) converted data in the computer memory. The improvement of $S / N$ ratio was proportional to the square root of the accumulation number. However, the obtained densitograms by this method showed distorted peaks compared with those obtained by s-PAS. Moreover, in FS-PAS measurements the accumulation number and the dynamic range were limited by the bit number of the $A / D$ converter used ( $8 \mathrm{bit}$ ). We describe in this paper some improvements of FS-PAS and its application to quantitative imaging of protein spots which have been two-dimensionally electrophoresed on polyacrylamide gels.

\section{Experimental}

Materials and method

Two-dimensional electrophoresis. Two dimensional electrophoresis was conducted according to a method by $\mathrm{O}^{\prime}$ Farrell ${ }^{3}$, with some modification. The equipment 
used included: a high-voltage regulator, isoelectric electrophoresis, slab-gel electrophoresis, and electroblotting; all equipment was made in our laboratory. A pH gradient rod gel $(0.75 \mathrm{~mm}$ i.d., $35 \mathrm{~mm}, \mathrm{pH} 3.5-10)$ was used for isoelectric focusing with $10 \mathrm{mM}$ phosphoric acid as an anode solution and $20 \mathrm{mM} \mathrm{NaOH}$ as a cathodic solution. The voltage to the apparatus was maintained constant at $400 \mathrm{~V}$. After isoelectric focusing, the gel was removed from the glass tube, and then put on a micro slab gel $(35 \times 40 \times 1 \mathrm{~mm})$ with a $7.5-20 \%$ linear gradient for sodium dodecylsulfate (SDS) gel electrophoresis. SDS gels were electrophoresed at a constant current $(10 \mathrm{~mA})$. The protein mixture was fractionated by this state according to the molecular weight. The SDS electrophoresis was completed when marker dye (Bromphenol Blue) was electrophoresed out from the end of the gel. After SDS electrophoresis was completed, the gel was removed from the glass plates and then attached to the blotting apparatus (reported by Towbin et al. ${ }^{12}$ ). Proteins on polyacrylamide gel were electrophoretically transferred onto a nitrocellulose sheet (Bio-Rad, pore size $0.45 \mu \mathrm{m}$ ) at a constant voltage $(20 \mathrm{~V})$. The gels were stained with Amidoblack 10B. The nitrocellulose sheet was fixed with $7 \%$ trichloroacetic acid and then stained with Amidoblack 10B. Excessive dye was destained with $2 \%$ acetic acid, $90 \%$ methanol solution. The protein on the nitrocellulose sheet was then dried in vacuo and stuck to a $40 \times 45 \times 1 \mathrm{~mm}$ glass plate with double-sided adhesive tape for a FS-PAS measurement.

Photoacoustic apparatus. The FS-PAS system was improved from that mentioned in a previous report to more precisely obtain a wide dynamic range photoacoustic signal. A photoacoustic cell $(42 \times 47 \times 3 \mathrm{~mm}$ in internal size) was moved by a slide table (Sigma, LT100s). The direction of the movement was at right angles to the laser-beam scan. The laser beam (Huges Aircraft Co. Model 3230-HPC, $P=15 \mathrm{~mW}$ ) was reflected by a moving aluminum-coated mirror (Sigma, $\lambda / 10)$ so as to scan the cell. The scanning mechanisms are shown in Fig. 1: (A) In the single-scan type, the mirror was directly connected to the shaft of a geared DC motor. (B) In the sinusoidal scan type, the mirror was moved by an offset DC motor through a crank

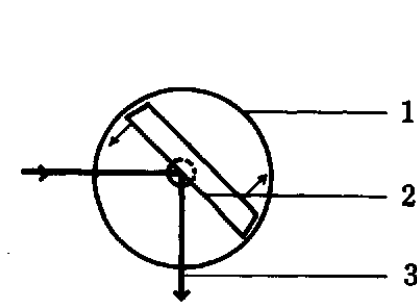

(A)

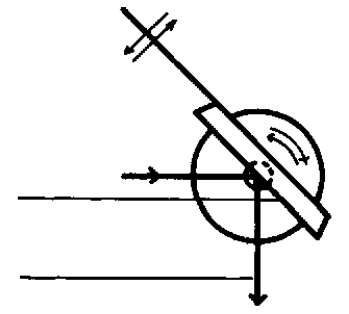

(B)
Fig. 1 Scan mechanisms of laser beam. (A) single scan mechanism; (B) sinusoidal scan mechanism. 1, DC geared motor; 2 , moving mirror; 3 , laser beam. shaft. The photoacoustic signal was amplified by a preamplifier (made by us and described later). When the laser beam triggered the photodiode, the signal initiated $\mathrm{A} / \mathrm{D}$ conversion by a 12-bit $\mathrm{A} / \mathrm{D}$ converter (Neolog Co. Model PC-2098) at a constant interval $(100$ or $200 \mu \mathrm{s})$. Digitalized data were transferred to computer memory (NEC, PC9801VM2) and stored on a floppy disk. The A/D conversion was stopped at a word length of $2 \mathrm{~K}$ words. The laser beam was usually scanned on the same lane for 4-25 times. These data were accumulated in computer memory and then divided by the accumulation number so as to improve the $S / N$ ratio.

To compare the performance of the FS-PAS system, a light scanning photoacoustic densitometer was used. The analytical conditions were as follows: time constant $=$ $3.0 \mathrm{~s}$, chopping frequency $=32 \mathrm{~Hz}$.

Chemicals. All chemicals used for the electrophoresis were reagent grade or for electrophoresis. To establish a pH gradient, ampholine (LKB, 3.5-10, 5-7.5) was used. Ovalbumin (Sigma, A-7642) and newborn calf serum (Gibco) were used as samples.

\section{Results and Discussion}

FS-PAS gives a photoacoustic signal as a sound wave, which is continuously detected by a microphone located at one end of a photoacoustic cell. The line scanned by the laser beam contributes to the signal generation; a chromatogram is, consequently, obtained during the scanned period $(0.1-0.5 \mathrm{~s})$. However, the obtained signal was usually accompanied by a depression peak after the sample peak. We have reported that this depression could mainly be attributed to the character of the preamplifier. ${ }^{13}$ When a sample was scanned by the single-scan-type mechanism (Fig. 1 (a)), which was used in previous work, the lowest frequency input into the preamplifier was determined by the interval between one scan and the next $(10-30 \mathrm{~s})$. On the other hand, during sinusoidal scans, the repeated frequency was determined by the cycle of the sinusoidal scan. Though only a qualitative discussion is given below, it covers the relationship between photoacoustic signals and the scan mechanism.

A single-scan-type model is summarized in Fig. 2(a).

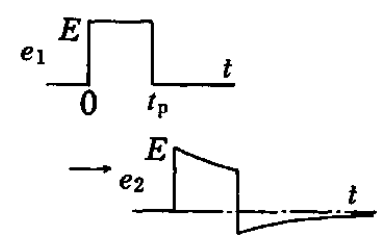

(A)

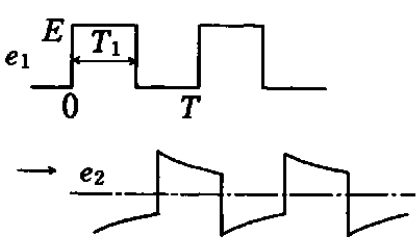

(B)
Fig. 2 Typical response of first order high pass filter. (A) isolated single pulse; (B) continuous pulses. $e_{1}$, waveform of input signal; $e_{2}$, typical waveform of output signal. 
Since the lowest frequency is determined by a large interval between scans $(10-30 \mathrm{~s})$, the signal obtained during a single scan can be thought of as an isolated single pulse. When a single pulse was introduced into a first-order high-pass filter (usually formed by a load resistor and a input capacitor), the observed output was distorted like Fig. 2(a). The output can be described as

$$
\begin{aligned}
e_{2} & =E \exp (-1 / \mathrm{X}) & & \left(0<t<t_{\mathrm{p}}\right) \\
& =E\left\{\exp (-t / X)-\exp \left[-\left(t-t_{\mathrm{p}}\right) / X\right]\right\} & & \left(t_{\mathrm{p}}<t\right)
\end{aligned}
$$

where $X$ is the time constant of the high-pass filter and $t_{\mathrm{p}}$ is the pulse width. The output signal is described in terms of transient response. On the other hand, when a sample was sinusoidally scanned by scan-type (b) (Fig. 1(b)), the lowest frequency was determined by the cycle of the sinusoidal scan. The output signal of the first-order high-pass filter observed with a sinusoidal scan is summarized in Fig. 2(b). The output of the preamplifier is described as

$$
\begin{aligned}
e_{2}= & e_{2}^{\prime}+\left(\text { repetition of } e_{2}^{\prime \prime} \text { at the cycle of } T\right) \\
e_{2}^{\prime}= & E \times \frac{1-\exp \left(T_{1} / X\right)}{1-\exp (T / X)} \times \exp (-t / X) \\
e_{2}^{\prime \prime}= & E \times \frac{1-\exp \left[-\left(T-T_{1}\right) / X\right]}{1-\exp (T / X)} \\
& \times \exp (-t / X) \\
= & -E \times \frac{1-\exp \left[-\left(T_{1} / X\right)\right]}{1-\exp (-T / X)} \\
& \times \exp \left[-\left(t-T_{1}\right) / X\right]
\end{aligned}
$$

$$
=0
$$

The $e_{2}^{\prime}$ term shows the transient response, which eventually reached zero. The signal observed in steady state is a repetition of $e_{2}^{\prime \prime}$ at a cycle of $T$. The distortion of the output signal in Fig. 2(b) was caused by the frequency response of the preamplifier; thus, a preamplifier with a Bessel high-pass filter type was used. This preamplifier was used to change a continuous rectangular DC pulse signal into an AC signal without distortion, when the repeated frequency was more than 10-times larger than the cut-off frequency of the Bessel filter. When the beam was sinusoidally scanned by scan-type 2, the signal could be amplified by a Bessel filter input preamplifier without distortion. We have constructed a Bessel filter input preamplifier to amplify a FS-PAS signal with a cut-off frequency of $0.01 \mathrm{~Hz}$. In this case, however, if the sinusoidal scan cycle was less than $0.1 \mathrm{~Hz}$, the signal was distorted by the preamplifier. Figure 3 shows a comparison of the photoacoustic image for carbon black on nitrocellulose sheet obtained
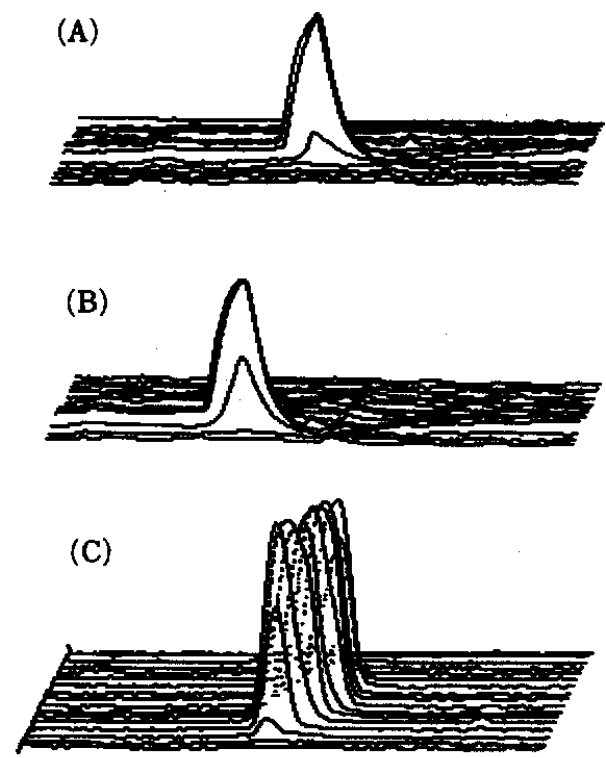

Fig. 3 Comparison of photoacoustic images of carbon black on nitrocellulose sheet. (A) sinusoidal scan; (B) single scan; (C) s-PAS.

by a single sacn (A), a sinusoidal scan (B) and conventional s-PAS (C). The sinusoidal scan coupled with a Bessel filter input-type preamplifier gave a smaller distortion than the single scan type. However, even in the improved method, the peak was accompanied by a small depression after the sample peak, compared with an s-PAS densitogram. It would be caused by the frequency response of the microphone itself and the reflection of heat waves at the cell wall. As a result of using this preamplifier and a sinusoidal laser-beam scan, the distortion caused by the characteristics of the preamplifier did not need to be taken into account for the densitogram obtained by the proposed method.

In the FS-PAS measurement used in this experiment, since the signal obtained by the laser beam scan was accumulated in computer memory so as to improve the $S / N$ ratio, any variation of the laser-beam scanning speed affected the accumulation error. When a signal was A/D converted every $100 \mu$ s and the beam scanned at $14 \mathrm{~cm} / \mathrm{s}$, the variation of the scanning speed $1 \%$ (RSD) correspond to a variation of 30 words at the word length of $1 \mathrm{~K}$ words. For the single-scan type used in this experiment the relative standard deviation of the scanning speed was below $0.75 \%$ at any scanning speed $(10-30 \mathrm{~cm} / \mathrm{s})$. In a sinusoidal scan, the RSD for the scanning speed was approximately $1.0 \%$.

Two-dimensional electrophoresis shows a very high resolution for complex mixtures of proteins. However, it is time consuming to acquire an electropherogram because of the large size of the gel. Two-dimensional micro-electrophoresis has many advantages over conventional size electrophoresis regarding detectability and the time to perform electrophoresis. Okuyama et al. ${ }^{14}$ have reported a two-dimensional micro-electro- 


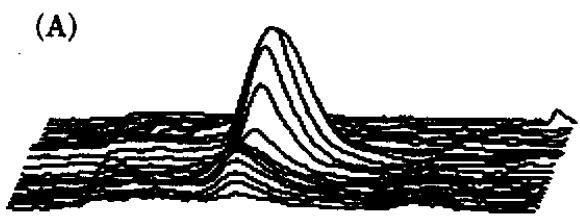

(B)

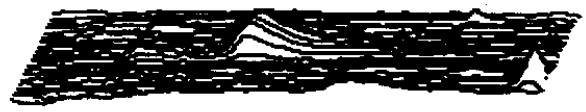

Fig. 4 Typical photoacoustic images of ovalbumin stained with Amidoblack 10B on nitrocellulose sheet (A) and on polyacrylamide gel (B).

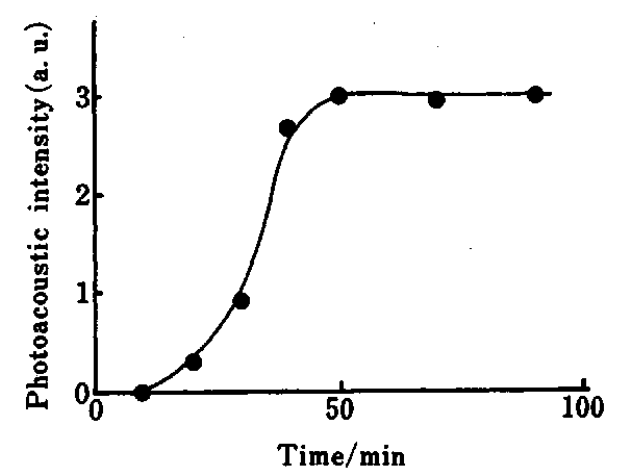

Fig. 5 Relationship between photoacoustic intensity for ovalbumin $2.04 \mu \mathrm{g}$ and electroblotting time from polyacrylamide gel (7.5-20\% linear gradient gel) to nitrocellulose sheet.

phoresis. We used a smaller size isoelectric focusing gel in capillary tubing $(0.75 \mathrm{~mm}$ i.d., $35 \mathrm{~mm}$ length). The conventional size isoelectric focusing rod gels usually require pre-electrofocusing in order to stabilize the $\mathrm{pH}$ gradient formed by the carrier ampholine. In our experiment preelectrophoresis had no notable effect on the separation. When a sample was electrofocused at $400 \mathrm{~V}$, sample migration seemed to reach a steady state after $20 \mathrm{~min}$. To complete isoelectric focusing, the gel was electrophoresed at $400 \mathrm{~V}$ for $30 \mathrm{~min}$. It was thought that this phenomenon is influenced by the size of the rod gel.

Generally, the photoacoustic signals obtained by sPAS or FS-PAS, depend upon the thermal characteristics and optical absorptivity of the sample. When an electrophoresed gel was directly stained with Amidoblack 10B, the signal obtained by fast laser-beam scanning was very weak. It is thought that because the polyacrylamide gel, which was previously dried in vacuo to form a film, was thermally thick and the sample proteins were located in a gel, the absorbed laser beam, which contributed to the photoacoustic signal, was very small. To obtain a larger signal, the proteins on a polyacrylamide gel were electrophoretically transferred

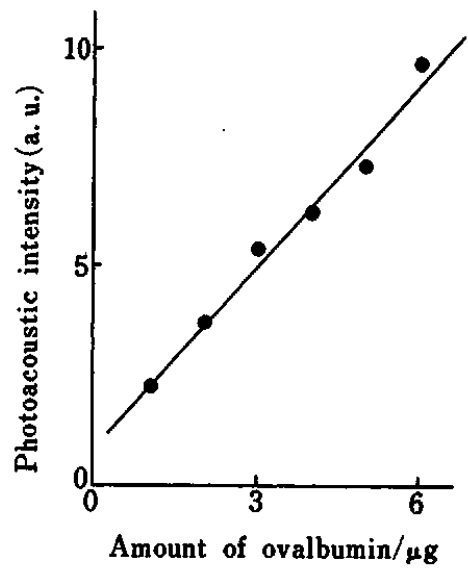

Fig. 6 Calibration curve for ovalbumin stained with Amidoblack $10 \mathrm{~B}$ on nitrocellulose sheet.

onto a nitrocellulose sheet. The thermal character of the sample was then represented by nitrocellulose in the place of polyacrylamide. Figure 4 shows a comparison of typical photoacoustic densitograms obtained by polyacrylamide gel and nitrocellulose sheet. Although the stained spot of sample ovalbumin $(2.04 \mu \mathrm{g})$ on polyacrylamide gel visually showed 2 - or 3-times greater contrast than on nitrocellulose sheets, the signal using a nitrocellulose sheet was about five-times greater than that by polyacrylamide gel. When the proteins on a polyacrylamide gel were transferred onto a nitrocellulose sheet, electrophoresis was performed in a direction perpendicular to the gel. Figure 5 shows the relationship between the electroblotting time at $20 \mathrm{~V}$ and the photoacoustic signal magnitude. The signal reached a maximum after $40 \mathrm{~min}$. Though proteins were not detected visually on polyacrylamide gel, with more than 120 min of blotting time the photoacoustic signal was decreased since, perhaps, they were electrophoresed out through a nitrocellulose sheet.

Figure 6 shows plots of the photoacoustic signal magnitude versus the amount of ovalbmin on nitrocellulose sheet which was electrophoresed and blotted, as described above. The signal magnitudes represented in the figure are the sum of the peak areas of each densitogram. The calibration curve obtained by the improved FS-PAS system shows a linear relationship from 1 to $6 \mu \mathrm{g}$. The correlation factor of the line was above 0.97 . The reproducibility (RSD) of measurements for ovalbumin on four different nitrocellulose sheets were 8 to $13 \%$. On the other hand, the reproducibility of four measurements for ovalbumin $(4.98 \mu \mathrm{g})$ on the same plate was below $5 \%$. The detection limit of ovalbumin was about $150 \mathrm{ng}(S / N=$ 2).

To confirm the performance of the proposed method, ovalbumin $(1.67 \mu \mathrm{g})$ was added to calf serum $(3.33 \mu \mathrm{g}$ as total protein) and the amount of ovalbumin was determined by this method. Throughout the procedure, the spot of ovalbumin was always isolated from calf 
Table 1 Recovery of ovalbumin spiked on calf serum

\begin{tabular}{|c|c|c|c|c|}
\hline \multirow{2}{*}{ Method } & \multicolumn{2}{|c|}{ OVA/ $\mu g$} & \multirow{2}{*}{$\begin{array}{c}\text { RSD, } \\
\%\end{array}$} & \multirow{2}{*}{$\begin{array}{c}\text { Recovery, } \\
\%\end{array}$} \\
\hline & Added & Found & & \\
\hline Single scan & 1.67 & 1.84 & 9.60 & 110.2 \\
\hline Sinusoidal scan & 1.67 & 1.73 & 13.0 & 103.6 \\
\hline Lowry's assay's & 1.67 & 1.67 & - & - \\
\hline
\end{tabular}

Ovalbumin (OVA) $1.67 \mu \mathrm{g}$ was spiked on $3.33 \mu \mathrm{g}$ of calf serum.

serum. Table 1 shows the results of a recovery test using both the single-scan and sinusoidal-scan types. In the single-scan mechanism, the determined value was greater than that using the sinusoidal scan. The large RSD of measurements can be attributed to the complex procedures from isoelectric focusing to the staining of proteins on nitrocellulose sheets. However, ovalbumin was determined at more than $100 \%$ using both methods. The reason why the value is so large seems to be due because the ovalbumin spot was integrated on the tailing line of a large spot of calf serum. The greater the resolution of the apparatus allows an improvement in the determination error. The resolution of the system is restricted by both the beam diameter on the sample surface and the frequency response of preamplifier. The frequency response of the Bessel high-pass filter-input type was in the range $0.1-1 \mathrm{kHz}(-1 \mathrm{~dB})$. The $1 \mathrm{kHz}$ frequency corresponded to $100 \mu \mathrm{m}$ in a chromatogram when the beam was scanned at $10 \mathrm{~cm} / \mathrm{s}$. Other experiments show that the resolution of this apparatus is about $1.5 \mathrm{~mm}$. The resolution could be much improved by focusing the laser beam with some appropriate lens.
We described here the applications of the FS-PAS for a quantitative imaging of electrophoresed protein on a nitrocellulose sheet. The potential sensitivity and speed of data acquisition provide FS-PAS with a wide range of applications in various biological fields.

\section{References}

1. A. Chrambach, Mol. Cell. Biochem., 29, 23 (1980).

2. U. K. Laemmli, Nature [London], 277, 680 (1970).

3. P. H. O'Farrell, J. Biol. Chem., 250, 4007 (1975).

4. G. T. Sterenson, Nature [London], 247, 477 (1974).

5. C. Fenner, R. R. Traut, D. T. Mason and J. WilkmanCoffelt, Anal. Biochem., 63, 595 (1975).

6. T. Manabe and T. Okuyama, J. Chromatogr., 264, 435 (1983).

7. U. Moller, H. P. Cost, S. Schneider and H. Koufal, Electrophoresis, 4, 148 (1983).

8. K. Imaeda, K. Ohsawa and K. Uchiyama, Bunseki Kagaku, 33, 320 (1984).

9. K. Imaeda, K. Ohsawa and K. Uchiyama, Bunseki Kagaku, 34, 346 (1985).

10. K. Imaeda, K. Ohsawa, K. Uchiyama, S. Nakamura and T. Tokieda, Anal. Sci., 2, 9 (1986).

11. K. Imaeda, K. Ohsawa, K. Uchiyama and S. Nakamura, Anal. Sci., 3, 11 (1987).

12. H. Towbin, T. Staehelin and J. Gordon, Proc. Natl. Acad. USA, 76, 4350 (1979).

13. K. Uchiyama, K. Ohsawa, Y. Yoshimura, K. Arai and K. Imaeda, Anal. Sci., 4, 675 (1988).

14. T. Kadoya, K. Hukumoto, H. Manabe and T. Okuyama, Bronseki Kagaku, 34, 151 (1985).

15. O. H. Lowry, N. J. Rosebrough, A. L. Farr and R. J. Randall, J. Biol. Chem., 193, 265 (1951).

(Received November 30, 1989) (Accepted March 12, 1990) 